\title{
Tibiofemoral wear in standard and non-standard squat: implication for total knee arthroplasty
}

\author{
Gusztáv Fekete ${ }^{1,2}$ \\ Dong Sun ${ }^{2}$ \\ Yaodong $\mathrm{Gu}^{2}$ \\ Patric Daniel Neis ${ }^{3}$ \\ Ney Francisco Ferreira $^{3}$ \\ Bernardo Innocenti ${ }^{4}$ \\ Béla M. Csizmadia ${ }^{5}$ \\ 1 Savaria Institute of Technology, Eötvös Loránd \\ University, Szombathely, Hungary \\ 2 Research Academy of Grand Health, Ningbo \\ University, Ningbo, China \\ ${ }^{3}$ Laboratory of Tribology, Federal University of Rio \\ Grande do Sul, Porto Alegre, Brazil \\ ${ }^{4}$ BEAMS Department, Université Libre de Bruxelles, \\ Brussels, Belgium \\ ${ }^{5}$ Institute of Mechanics and Machinery, Szent István \\ University, Gödöllő, Hungary
}

Corresponding author:

Gusztáv Fekete

Savaria Institute of Technology, Faculty

of Informatics,

Eötvös Loránd University, Szombathely, Hungary

E-mail: fg@inf.elte.hu

\section{Summary}

Introduction: Due to the more resilient biomaterials, problems related to wear in total knee replacements (TKRs) have decreased but not disappeared. In the design-related factors, wear is still the second most important mechanical factor that limits the lifetime of TKRs and it is also highly influenced by the local kinematics of the knee. During wear experiments, constant load and slide-roll ratio is frequently applied in tribo-tests beside other important parameters. Nevertheless, numerous studies demonstrated that constant slide-roll ratio is not accurate approach if TKR wear is modelled, while instead of a constant load, a flexion-angle dependent tibiofemoral force should be involved into the wear model to obtain realistic results.

Methods: A new analytical wear model, based upon Archard's law, is introduced, which can determine the effect of the tibiofemoral force and the varying slide-roll on wear between the tibiofe- moral connection under standard and non-standard squat movement.

Results: The calculated total wear with constant slide-roll during standard squat was 5.5 times higher compared to the reference value, while if total wear includes varying slide-roll during standard squat, the calculated wear was approximately 6.25 times higher. With regard to non-standard squat, total wear with constant slide-roll during standard squat was 4.16 times higher than the reference value.

If total wear included varying slide-roll, the calculated wear was approximately 4.75 times higher.

Conclusions: It was demonstrated that the augmented force parameter solely caused $65 \%$ higher wear volume while the slide-roll ratio itself increased wear volume by $15 \%$ higher compared to the reference value. These results state that the force component has the major effect on wear propagation while non-standard squat should be proposed for TKR patients as rehabilitation exercise.

KEY WORDS: analytical model, rehabilitation, slide-roll ratio, squat, tibiofemoral force, wear.

\section{Introduction}

Based on the new results of researchers and the technological advances of the manufacturers, more effective, affordable and reliable total knee replacements (TKRs) are provided to patients every year. It must be noted that the contemporary TKRs can last more than two decades ${ }^{1}$, however failures cannot be completely eliminated. Sharkey et al. pointed out how quickly the trend of TKR failures changed in the past 10 years and moved to the direction of loosening, infection, instability, arthrofibrosis and periprosthetic fracture, while wear in the polyethylene parts became only the seventh most damaging factor in the line ${ }^{2}$. In general, wear in implants is in one hand caused by the non-congruent form of the knee joint together with its natural instability. On the other hand, emerging particle debris is also a relevant cause of cartilage damage. It is worth noting that the phenomenon of wear is in relation with several interrelated factors, thus it must be examined as a system not as a material property ${ }^{3}$. Even though wear is not considered nowadays as the most imminent problem in TKR failure, it still stands as the second most relevant me- 
chanical factor, after periprosthetic fracture, that limits lifetime of $\mathrm{TKRs}^{4,5}$. It is also highly influenced by the kinematics of the knee joint6.

Besides the two most important parameters in wear studies, the load and the length of sliding, slide-roll ratio is also frequently taken into account when tribological tests are carried out on pin-on-disc test rigs or knee simulators ${ }^{7,8}$. During wear tests, in most cases, a constant load is applied together with a constant slide-roll ratio based on the study of McGloughlin and Kavanagh ${ }^{9}$ and Hollman et al. ${ }^{10}$. It must be mentioned that the constant slide-roll approximation is correct if it is applied on pin-on-disc tests or ball-ondisc tests, where the kinematics of a simple contactpair is precisely adjusted to this special configuration. Nevertheless, several Authors published results against the idea of applying constant slide-roll ratio in case of TKRs ${ }^{11,12}$.

They referred to the complex geometry during contact, which highly alters the local kinematics, making the constant slide-roll approach unsuitable in TKR modelling.

It was also demonstrated in the study of Laurent et al. ${ }^{13}$ that wear mechanism is highly dependent not only on the load in the contact but also on the interfacial contact kinematics. This kinematics include a cyclic multidirectional path of motion and the slide-roll ratio as well.

In tribological experiments, it is a common main goal to determine the so-called specific wear rate regarding a predefined configuration of material, load and sliding length. These parameters are not constant in reality, however it is still commonly applied this way.

The aim of this paper is to unfold the effect of two new parameters, the tibiofemoral force representing the load and the slide-roll ration, in the phenomenon of wear. To reach this aim, a novel mathematical model is introduced on wear propagation between the tibiofemoral contacts. The load is no longer constant but incorporates the friction force, derived from the tibiofemoral force of the knee joint in the contact, while the length of sliding is complemented with a varying slide-roll ratio. For the analysis, a commonly applied wear model, Archard's law is used ${ }^{14}$, with the abovementioned augmentation. The method also shows the individual effect of the introduced parameters so in a tribological test rig it could be also examined.

In contempt of its simplicity, Archard's law is widely used in the contemporary literature ${ }^{15,16}$ and the predicted wear is reasonable agreement with other more sophisticated models like the model of Turell et al. ${ }^{17}$ or the model of Abdelgaied et al. ${ }^{18}$. Squat is chosen for analyses with regard to wear, since if this motion is carried out then the highest contact forces ${ }^{19}$ and the highest slide-roll ratio can be experienced ${ }^{12}$. As mentioned earlier, squat is a widely exercised movement for rehabilitation and for thigh muscles strengthening due to sport or medical reasons ${ }^{20,21}$, therefore it is important to know the aspect of wear with regard to this motion.

\section{Methods}

In this section, a new analytical model is introduced. In this model, two components, the load and the sliding distance will be further extended by additional biomechanical factors with regards to the knee joint. It must be stated that the model deals with a singlepoint-contact, thus in this present form it cannot discriminate wear between the medial and the lateral compartment.

\section{Wear modelling}

The instantaneous volume of material removed from the tibiofemoral surface of the TKR due to mild wear is predicted by Archard's law ${ }^{14}$ :

$$
d V=k \cdot F_{N} \cdot d s
$$

where $k$ is the specific wear rate $\left[\mathrm{mm}^{3} / \mathrm{Nm}\right.$ ] which is a material dependent quantity, $F_{N}$ is the normal force [N] acting between the contact surfaces of the tibia and the femur, while $d s$ is the infinitesimal length of sliding [m].

There are two parameters in this equation that are to be further enhanced: the force and the sliding length. In this study, the wear mechanism between the connecting surfaces is supposed to be abrasive based (two-body). This means that during contact, the harder metallic femoral part ploughs into the softer polyethylene tibial part, and in the connecting point a reaction force appears (Fig. 1). The first step is to approximate this force with the Coulomb-law:

$$
F_{\text {friction }}=\mu_{k} \cdot F_{N}
$$

Where $\mu_{k}$ is the coefficient of kinetic friction.

In abrasive wear mechanism, frictional component is responsible for creating a shear stress in the upper surface of the material which begins losing small debris. Therefore, it will certainly become a more precise approximation if the friction force, deduced from the tibiofemoral force, is introduced in the wear equation:

$$
d V=k \cdot F_{\text {friction }} \cdot d s=k \cdot \mu_{k} \cdot F_{N} \cdot d s
$$

\section{Force modelling for wear equation}

The normal force $F_{N}$ in Equation 3 that acts between the connecting surfaces is considered constant during wear tests. Nevertheless, in case of the tibiofemoral connection during squat movement, this force is a flexion angle dependent quantity and it is called tibiofemoral force. It has been deduced analytically for standard (no horizontal movement of the center of gravity is permitted) and non-standard ${ }^{19}$ (horizontal movement of the center of gravity is allowed as it is in real life) squat motion (Fig. 2), which makes it an adequate choice to incorporate this force into the new model. The function of the tibiofemoral force can be described as follows ${ }^{19}$ : 


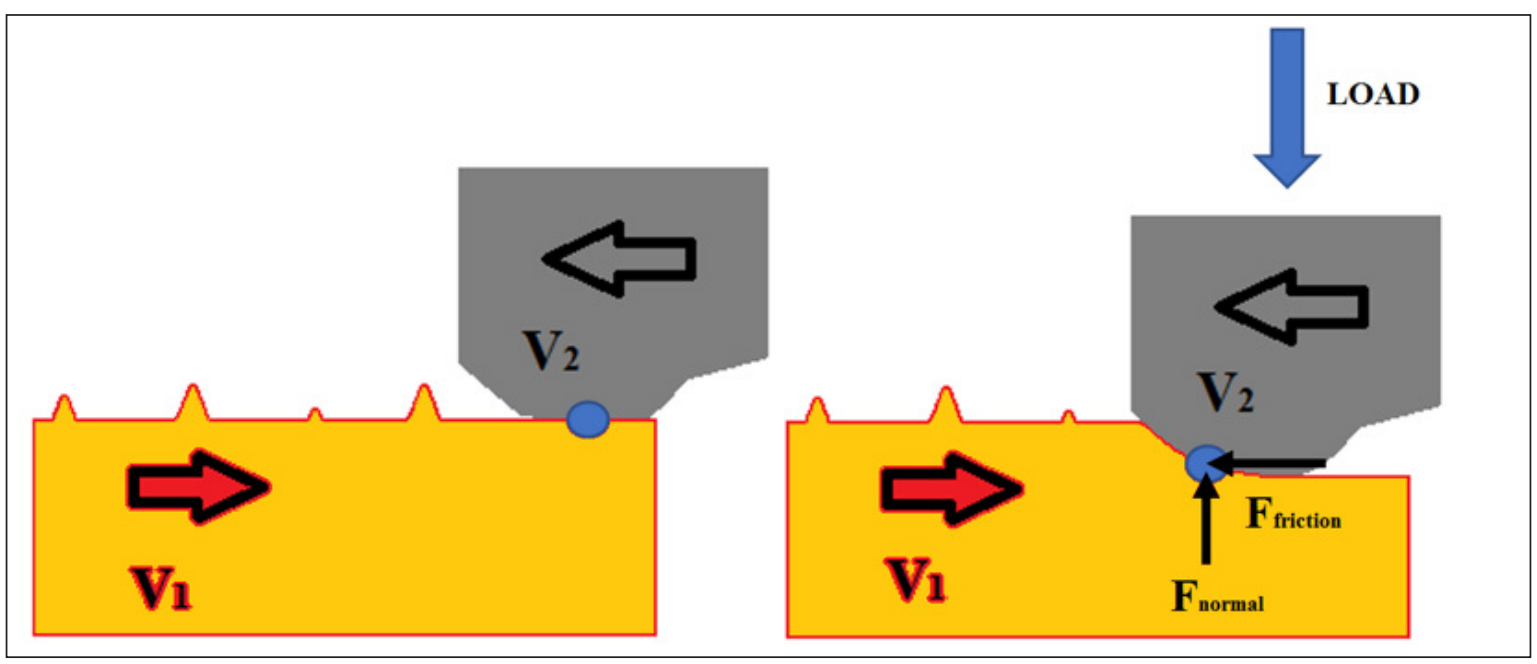

Figure 1. Two-body abrasive wear between the connecting surfaces.

$$
\frac{F_{t f}(\alpha)}{B W}=\frac{\lambda_{1}(\alpha) \cdot \sin \gamma(\alpha)}{\lambda_{p} \cdot \sin \beta(\alpha)+\lambda_{t} \cdot \cos \beta(\alpha)} \cdot\left(\frac{\cos \beta(\alpha)}{\cos \varphi(\alpha)}+\frac{\cos \gamma(\alpha)}{\cos \varphi(\alpha)}\right)(4)
$$

While the description of the ()-function ${ }^{19}$ :

$$
\varphi(\alpha)=\operatorname{arctg}\left[\frac{\left(\lambda_{1}(\alpha)-\lambda_{p}\right) \cdot \operatorname{tg} \beta(\alpha)-\lambda_{t}}{\lambda_{1}(\alpha) \cdot \operatorname{tg} \gamma(\alpha)+\lambda_{p} \cdot \operatorname{tg} \beta(\alpha)+\lambda_{t}} \cdot \operatorname{tg} \gamma(\alpha)\right]
$$

The parameters of the equation are summarized in Table I.

If Equation 4 is multiplied by an arbitrary chosen bodyweight (BW) then the tibiofemoral force is obtained. In the above mentioned analytical expressions, $\lambda_{1}$ function represents the horizontal movement of the center of gravity during squat. If the linear function is used as given in Table I, then a more realistic, so-called non-standard squat is carried out where the torso leans forward and it helps the knee to release a considerable amount of moment (Fig. 3 right drawing). This is the squat type that was practiced by most people in everyday life. The so-called standard squat was defined for tests and simple mechanical modeling, nonetheless, it does not consider the movement of the torso during the motion. Since higher knee moment, tibio- and patellofemoral forces act in the knee if this motion is exerted, it is not advised as a rehabilitation exercise.

In this study, both motions, therefore two types of tibiofemoral forces, are analyzed with their impact on wear. When standard squat is being examined the 1 function is constant with the value of 1 . This means that the torso does not lean forward (Fig. 3, left drawing).

In the augmented wear equation, the tibiofemoral force is included as follows:

$$
d V=k \cdot F_{\text {friction }} \cdot d s=k \cdot \mu_{k} \cdot F_{t f}(a) \cdot d s
$$

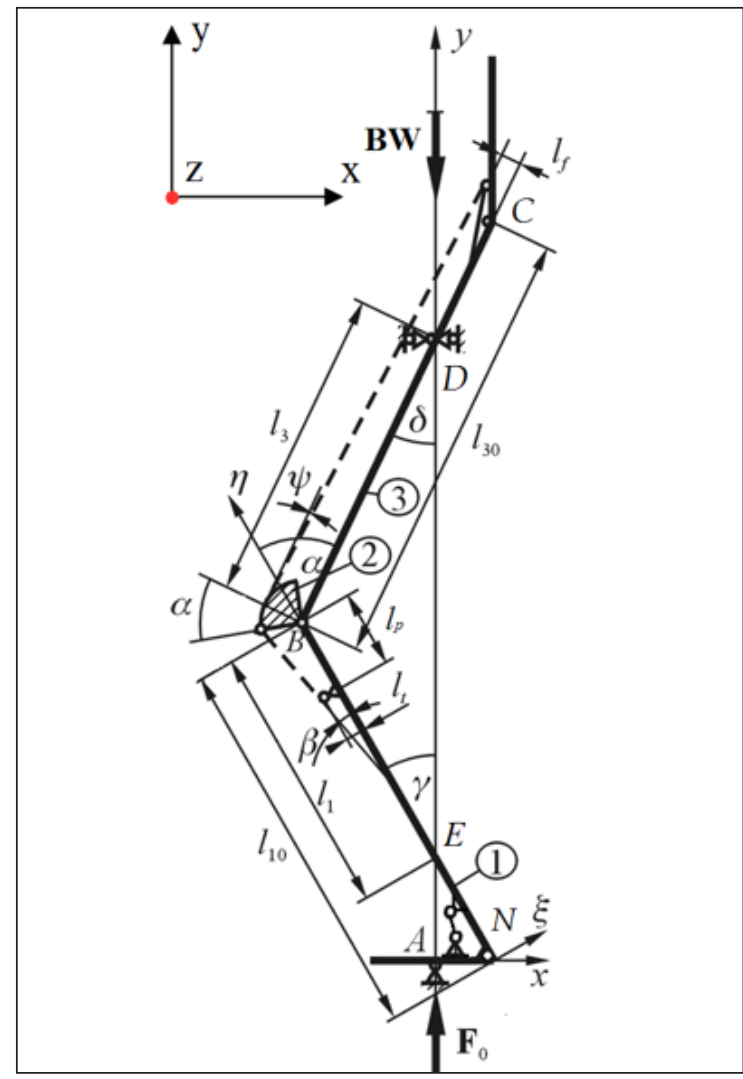

Figure 2. Mechanical model of squat with horizontally moving center of gravity.

If the tibiofemoral force is indexed as $F_{t f-n s t . C o G}$ then the horizontal movement of the center of gravity is also included, while with the index of $F_{t f-s t . C o G}$, the forward lean of the torso, the horizontal motion of the center of gravity, is excluded. 
Table I. Parameters of the tibiofemoral force and $\varphi$ function ${ }^{19}$.

\begin{tabular}{llll}
\hline Description & Parameter & Quantity or function & SD \\
\hline Dimensionless, intersected tibia length function & $\lambda_{1}(\alpha)=1_{1} / 1_{10}$ & $0.0024 \cdot \alpha+0.492[-]$ & 0.15 \\
Angle between the axis of tibia and the patellar tendon & $\varnothing(\alpha)$ & $-0.3861 \cdot \alpha+26.56\left[{ }^{\circ}\right]$ & 14 \\
Relative angle between the axis of tibia and the line of action of $B W$ & $\varnothing(\alpha)(\varnothing=\gamma / \alpha)$ & $-0.0026 \cdot \alpha+0.567[-]$ & 0.081 \\
Dimensionless thickness of shin & $\lambda_{t}=1_{t} / 1_{10}$ & $0.11[-]$ & 0.018 \\
Dimensionless thickness of thigh & $\lambda_{p}=1_{p} / 1_{30}$ & $0.1475[-]$ & 0.043 \\
\hline
\end{tabular}

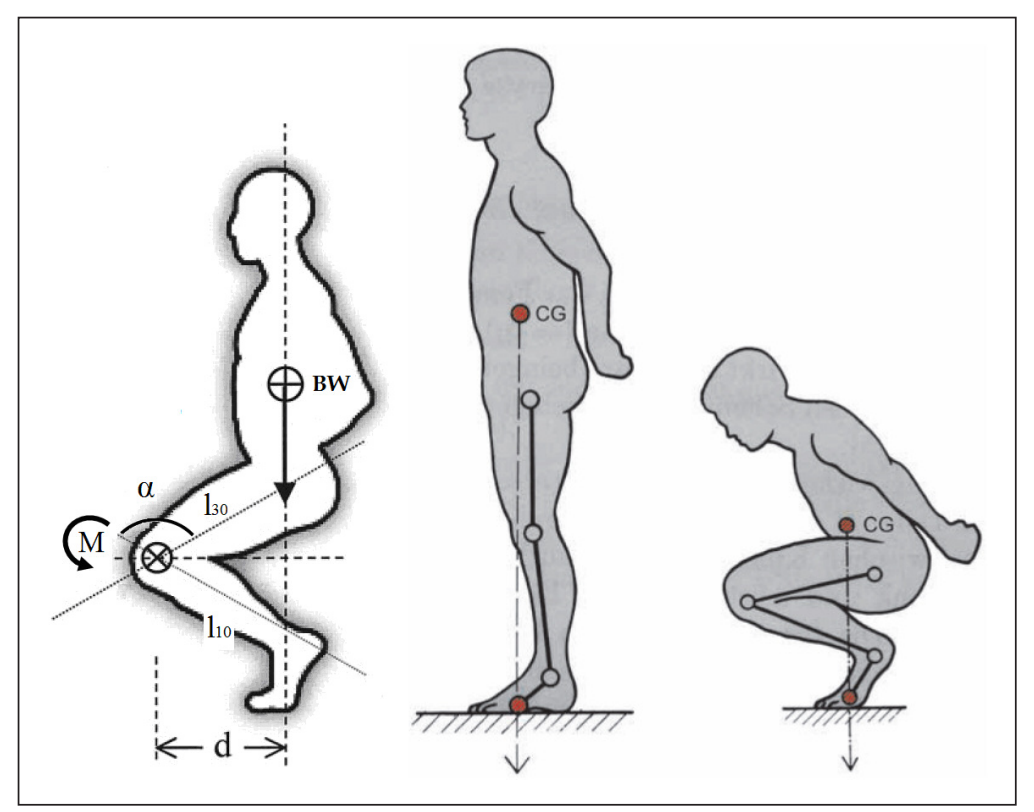

Figure 3. Standard (non-moving CoG) and non-standard (moving CoG) squatting.

\section{Slide/Roll modelling for wear equation}

Slide-roll is known to be an important parameter in wear modeling, however its quantitative effect has not been studied earlier. By having derived ${ }^{12}$ the slide-roll function, exact conclusions can be drawn about the features of the motion. If the ratio is zero then pure rolling is present, while one describes pure sliding. If the ratio is between zero and one, the movement is characterized as partial rolling and sliding. For example, a sliding-rolling ratio of 0.4 means $40 \%$ of sliding and $60 \%$ of rolling.

Fekete et al. ${ }^{12}$ introduced the slide-roll ratio by the use of arc lengths on both connecting bodies as follows:

$$
S / R(t)=\frac{\Delta s_{\text {tibia }}(t)-\Delta s_{f e m u r}(t)}{\Delta s_{\text {tibia }}(t)}
$$

where,

$$
\begin{aligned}
& \Delta s_{\text {femur }}(t)=s_{\text {femur }}(t)-s_{\text {femur }}(t-\Delta t) \\
& \Delta s_{\text {tibia }}(t)=s_{\text {tibia }}(t)-s_{\text {tibia }}(t-\Delta t)
\end{aligned}
$$

are the corresponding incremental differences of the connecting arc lengths.

A positive ratio shows the slip of the femur compared to the tibia. By this definition the Author derived an averaged slide-roll ratio with a standard deviation (SD $=0.136$ ) based on several TKRs as function of flexion angle ${ }^{12}$ :

$S / R(\alpha)=-5.16 \cdot 10^{-7} \cdot \alpha^{3}+1.235 \cdot 10^{-4} \cdot \alpha^{2}-4.113 \cdot 10^{-3} \cdot \alpha+0.226(8)$

This function is used for further calculations, and it is assumed that the function is applicable for flexion and extension movement as well.

With respect to the slide-roll ratio, there is an accordance between Authors that sliding is not dominant up to $65-67$ degree of flexion angle ${ }^{9-10}$, which practically means the domain of gait swing.

Nägerl et al. ${ }^{11}$ and Fekete et al. ${ }^{12}$ derived lower ratios between 0 and 65-67 flexion angle (Fig. 4), and above this region they presume that the slide-roll ratio can reach $95 \%$ of sliding as well ${ }^{11}$.

The interpretation why these ratios have such discrepancies are likely to be credited to the simplifications, e.g. frictionless contact, two-dimensional, simple circle or simplified knee geometry that the earlier Authors applied in their models ${ }^{9-10}$. Nägerl et al. ${ }^{11}$ 


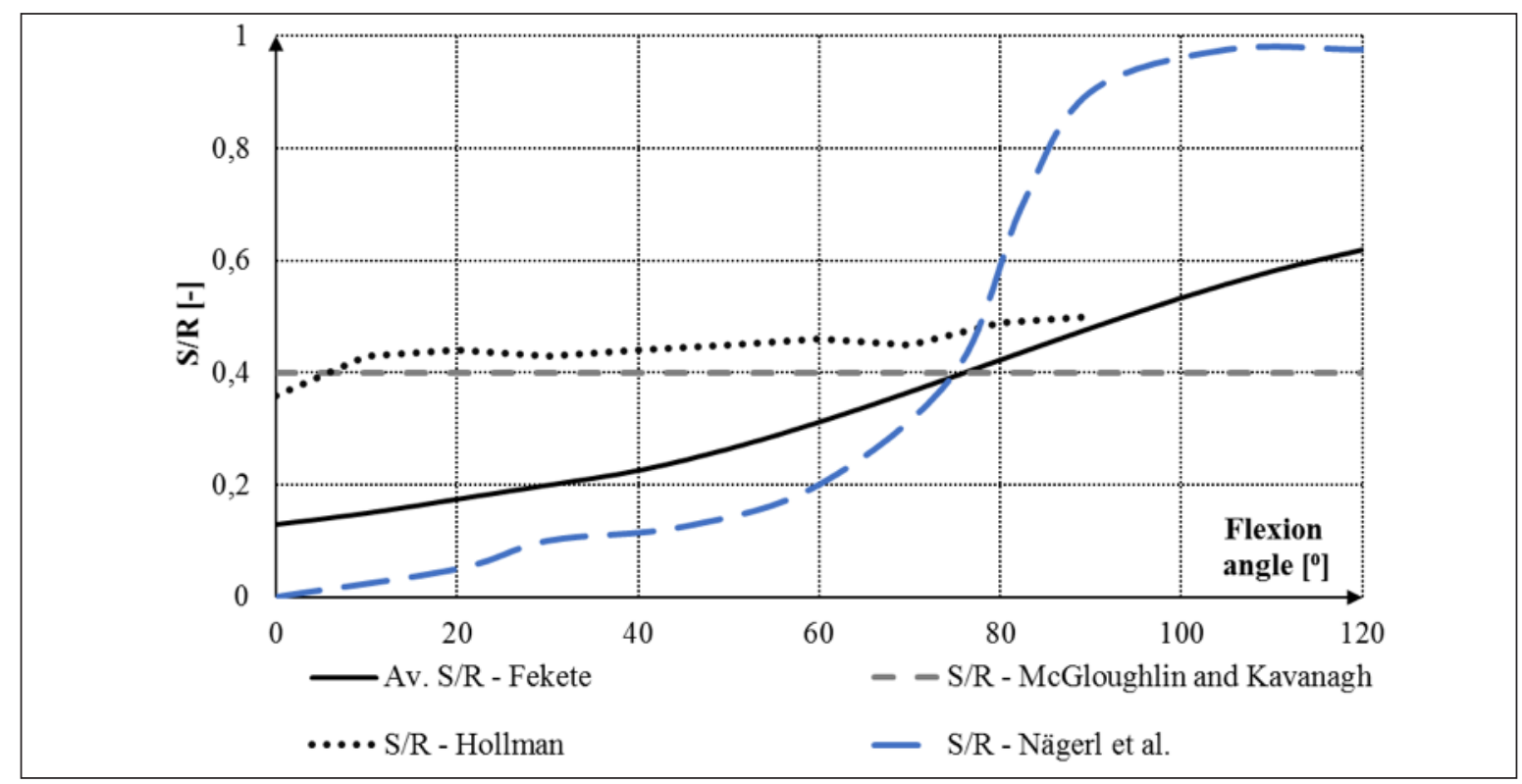

Figure 4. Summarized slide-roll ratios from different Authors.

used the first-time three-dimensional geometry to derive slide-roll ratio, while beside three-dimensionality, Fekete et al. ${ }^{12}$ included the effect of friction in their three-dimensional models.

Due to these enhancements, the latter two authors revealed non-linear phenomenon in the trend of the slide-roll ratio. Since slide-roll is known, it must be involved in the wear calculation to quantify its effect. To include the slide-roll ratio into the equation, let us the length of sliding rewrite as the product of sliding velocity $(\mathrm{m} / \mathrm{s})$ and infinitesimal time $(\mathrm{sec})$.

$d V=k \cdot \mu_{k} \cdot F_{t f}(\alpha) \cdot v_{\text {sliding }}(\alpha) \cdot d t$

The definition of slide-roll according to Fekete et al. ${ }^{12}$ can be expressed by the tangential velocities instead of the arc lengths:

$$
S / R(t)=\frac{v_{C T t}(t)-v_{C F t}(t)}{v_{C T t}(t)}=\frac{v_{\text {sliding }}(t)}{v_{C T t}(t)}
$$

Where the $v_{C T t}$ and $v_{C F t}$ are the tangential velocities in the instantaneous contact points of the tibia and the femur respectively. The difference of these velocities $\left(v_{C T t}-v_{C F t}=v_{\text {sliding }}\right)$ provides the so-called sliding velocity. If Equation 10 is rearranged to $v_{\text {sliding }}$ then it can be included into the augmented Archard's law is obtained:

$$
d V=k \cdot \mu_{k} \cdot F_{t f}(a) \cdot v_{C T t} S / R(t) \cdot d t
$$

$v_{C T t}$ velocity is kept constant similar to a wear test parameter $^{7}$ as an approximation. The original function of $F_{t f}$ is a function of flexion angle ${ }^{19}$, which can be transformed into time domain. This has been carried out in the study of Fekete et al. ${ }^{12}$, where the data were obtained in time domain during the squat simulations.
Thus, the final equation is obtained as shown below:

$$
d V=k \cdot \mu_{k} \cdot B W \cdot F_{t f}(t) \cdot v_{C T t} \cdot S / R(t) \cdot d t
$$

The parameters for Equation 12 are summarized in Table II.

In the followings, six total wear volumes were calculated, each of them, during one squat cycle. One cycle in squat is defined between 0 to 120 degrees of flexion and extension while the complete range where the propagation of wear monitored is 15 years. A usual knee wear simulation period is 3.5 million cycles $^{22,23}$ however squat and deep squat is less frequently exercised in everyday life.

If 3.5 million cycles were completed in 15 years, it would be 640 complete deep squat cycles carried out per day. This number of cycles is clearly overestimated, thus 50 deep squats per day will be assumed in this paper.

The total wear in one cycle can be calculated by integrating the wear functions over time. During deep squat, the total cycle is expanded to 120 degrees of flexion and extension angle where the time interval goes from 0 to 3.06 second during the squat motion (Fig. 5).

The total wear volumes are calculated in the following way: firstly, the slide-roll ratio is kept constant with a number of 0.4 according to the study of McGloughlin and Kavanagh ${ }^{9}$ together with Hollman et al. ${ }^{10}$ while a standard squat is carried out (the tibiofemoral force does not have the effect of the horizontally moving center of gravity). Secondly, the slide-roll ratio varies according to the function of Fekete et al. ${ }^{12}$ while a standard squat is carried out as well. Thirdly, the slide-roll ratio is kept constant with a number of 0.4 , but non-standard squat is considered (horizontally moving center of gravity), while fourthly, the slide-roll ratio varies during the non-standard squat. 
Table II. Wear parameters.

\begin{tabular}{ll}
\hline Parameter & Quantity or function \\
\hline$k$ : specific wear rate & $1.3 \cdot 10^{-6}\left[\mathrm{~mm}^{3} / \mathrm{Nm}\right]$ \\
$\mu_{k}:$ coefficient of kinetic friction ${ }^{12}$ & $0.003[-]$ \\
$F_{t f} / \mathrm{BW}:$ tibiofemoral force & $0.7702 \cdot \mathrm{t}^{3}+0.554 \cdot \mathrm{t}^{2}+1.6244 \cdot \mathrm{t}+1.0311[-]$ \\
relative to the body weight (moving CoG) & \\
$F_{t f} / \mathrm{BW}:$ tibiofemoral force relative to the body weight & $0.6652 \cdot \mathrm{t}^{3}+0.4397 \cdot \mathrm{t}^{2}+3.262 \cdot \mathrm{t}+1.0564[-]$ \\
(non-moving CoG) & $-0.111667 \cdot \mathrm{t}^{3}+0.3631732 \cdot \mathrm{t}^{2}+0.02378365 \cdot \mathrm{t}+1.137558[-]$ \\
$S / R:$ Varying slide-roll ratio & $0.4[-]$ \\
$S / R:$ Constant slide-roll ratio & $1000[\mathrm{~N}]$ \\
$B W:$ Body weight & $30[\mathrm{~mm} / \mathrm{s}]$ \\
$v_{C T t}:$ tangential velocity of the tibia in the contact & during squat: $1.53[\mathrm{~s}]$ \\
$t$ : duration of motion &
\end{tabular}

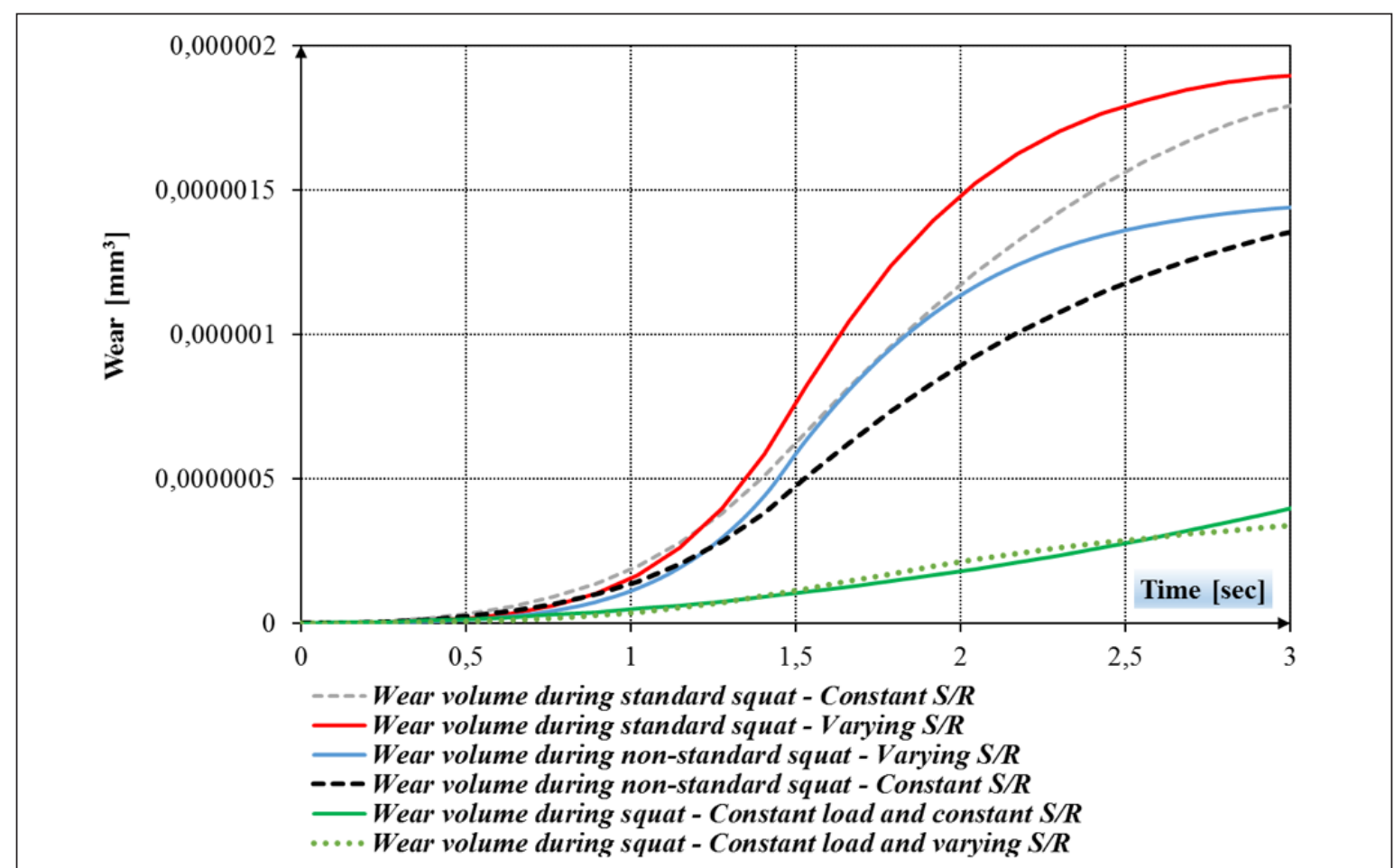

Figure 5. Wear propagation of the different models.

In In the fifth and sixth calculations, similarly to a wear test on a pin-on-disc or ball-on-disc configuration, the slide-roll is kept constant 0.4 in one case, while in the other case the ratio varies according to the function of Fekete et al. Regarding the force, only the simple Coulomb-law $\left(F_{s}=\mu_{k} \cdot F_{N}\right)$ is considered which results a constant load. The total wear during one squat cycle can be calculated as follows:

$\int_{V_{0}}^{V_{t o t a l}} d V=\int_{t_{0}}^{T} k \cdot \mu_{k} \cdot B W \cdot F_{t f}(t) \cdot v_{C T t} \cdot S / R(t) \cdot d t$
$V_{\text {total }}=\int_{t_{0}}^{T} k \cdot \mu_{k} \cdot B W \cdot F_{t f}(t) \cdot v_{C T t} \cdot S / R(t) \cdot d t+V_{0}$

In the first calculations, both models use the tibiofemoral force where the horizontal movement of the center of gravity is included (non-standard squat "nst"). Thus the total wear with varying slide-roll ratio:

$V_{n s t .-v . S / R}=\int_{0}^{3.06} k \cdot \mu_{k} \cdot B W \cdot F_{t f-n s t . C o G}(t) \cdot v_{C T t} \cdot S / R(t) \cdot d t=2.04 \cdot 10^{-6}$ 
$\left[\mathrm{mm}^{3} /\right.$ cycle $]$

While the total wear with constant slide-roll ratio:

$V_{n s t .-c . S / R}=\int_{0}^{3.06} k \cdot \mu_{k} \cdot B W \cdot F_{t f-n s t . C o G}(t) \cdot v_{C T t} \cdot S / R \cdot d t=1.76 \cdot 10^{-6}$

[mm³/cycle]

In the second calculations, both models use the tibiofemoral force where the center of gravity does not move horizontally (standard squat "st"). Total wear with varying slide-roll ratio but without the horizontal movement of the center of gravity:

$V_{s t .-v . S / R}=\int_{0}^{3.06} k \cdot \mu_{k} \cdot B W \cdot F_{t f-s t . C o G}(t) \cdot v_{C T t} \cdot S / R(t) \cdot d t=2.68 \cdot 10^{-6}$

$\left[\mathrm{mm}^{3} /\right.$ cycle]

While total wear with constant slide-roll ratio:

$V_{\text {st. }- \text { c.S } / R}=\int_{0}^{3.06} k \cdot \mu_{k} \cdot B W \cdot F_{t f-s t . C o G}(t) \cdot v_{C T t} \cdot S / R \cdot d t=2.33 \cdot 10^{-6}$

$\left[\mathrm{mm}^{3} /\right.$ cycle]

Total wear if constant load (BW) and constant slideroll ratio is used:

$V_{c . F+c . S / R}=\int_{0}^{3.06} k \cdot \mu_{k} \cdot B W \cdot v_{C T t} \cdot S / R \cdot d t=0.4289 \cdot 10^{-6}$

(19)

[mm³/cycle]

And at last, total wear if constant load (BW) and variable slide-roll ratio is used:

$V_{C . F+v . S / R}=\int_{0}^{3.06} k \cdot \mu_{k} \cdot B W \cdot v_{C T t} \cdot S / R(t) \cdot d t=0.4295 \cdot 10^{-6}$

(20)

[mm³/cycle]

\section{Results}

The calculations of the integrals have been summarized in Table III, where the percentile difference of the new parameters can be observed. By looking at the calculation of the wear test condition, only $1 \%$ difference is observable if varying or constant slide-roll ratio is applied. Therefore, the number of $0.429 \cdot 10^{-6}$ [ $\mathrm{mm}^{3} /$ cycle] can be chosen as a point of reference. When the results from the standard and non-standard squat are compared to the reference value, it is visible that wear, generated by the augmented models, is estimated 4.16-6.25 times more compared to the reference value. The significant difference is due to the new parameters, the varying tibiofemoral force function and slide-roll ratio.

As for quantitative conclusions, total wear with constant slide-roll during standard squat $\left(V_{\text {const.S/R }}\right)$ is 5.5 times higher compared to the reference value $\left(V_{\text {w.test }}\right)$, while if total wear includes varying slide-roll during standard squat, then the calculated wear is approximately 6.25 times higher.

With regards to non-standard squat, total wear with constant slide-roll during standard squat $\left(V_{\text {const.S/R }}\right)$ is 4.16 times higher compared to the reference value $\left(V_{\text {W.test }}\right)$, while if total wear includes varying slide-roll, then the calculated wear is approximately 4.75 times higher.

In case the wear model is coupled with constant slide-role ratio under non-standard squatting while compared to a wear model where the slide-roll ratio varies and standard squat is considered then $65 \%$ higher wear volume can be expected regarding the model with standard squat movement.

The propagation of wear for the lifetime cycle has been calculated as well, as it is seen in Figure 6.

Evidently, in case the wear test model is considered, where the load is constant, the different slide-roll ratio types (constant or varying) have negligible effect on wear in long term.

\section{Discussion}

It is clear that the highest wear volume is generated in case of standard squat due to the fact that the tibiofemoral force, without the effect of the horizontal lean of the torso, will reach the highest magnitude compared to other squatting motions. This makes the tibiofemoral force probably the most dominant parameter in the augmented Archard's law. According to

Table III. Wear results and comparison.

\begin{tabular}{|c|c|c|c|c|c|}
\hline \multirow{2}{*}{\multicolumn{2}{|c|}{$\begin{array}{l}\text { Wear volume } \\
{\left[10^{-6} \mathrm{~mm}^{3} / \mathrm{cycle}\right]}\end{array}$}} & \multirow{2}{*}{$\frac{\text { Standard squat }}{\frac{\text { rconst.S } / R^{\prime}}{V_{W . t e s t}}}$} & \multicolumn{3}{|c|}{ Non-standard squat } \\
\hline & & & vary.S/R & ${ }^{\prime}$ const.S/R & $v_{\text {vary.S } S / R}$ \\
\hline \multirow{2}{*}{$\begin{array}{l}\text { Wear test } \\
\text { condition }\end{array}$} & $V_{c F+c . S / R}=0.4289$ & & & & \\
\hline & $V_{c . F+v . S / R}=0.4295$ & & & & \\
\hline \multirow{2}{*}{$\begin{array}{l}\text { Standard } \\
\text { squat }\end{array}$} & $V_{\text {st. }-v . S / R}=2.68$ & & & & \\
\hline & $V_{\text {st. }- \text { c.S } / R}=2.33$ & & & & \\
\hline \multirow{2}{*}{$\begin{array}{l}\text { Non-standard } \\
\text { squat }\end{array}$} & $V_{\text {nst. }-v . S / R}=2.04$ & 5.5 & 6.25 & 4.16 & 4.75 \\
\hline & $V_{\text {nst.-c.S/R }}=1.76$ & & & & \\
\hline
\end{tabular}




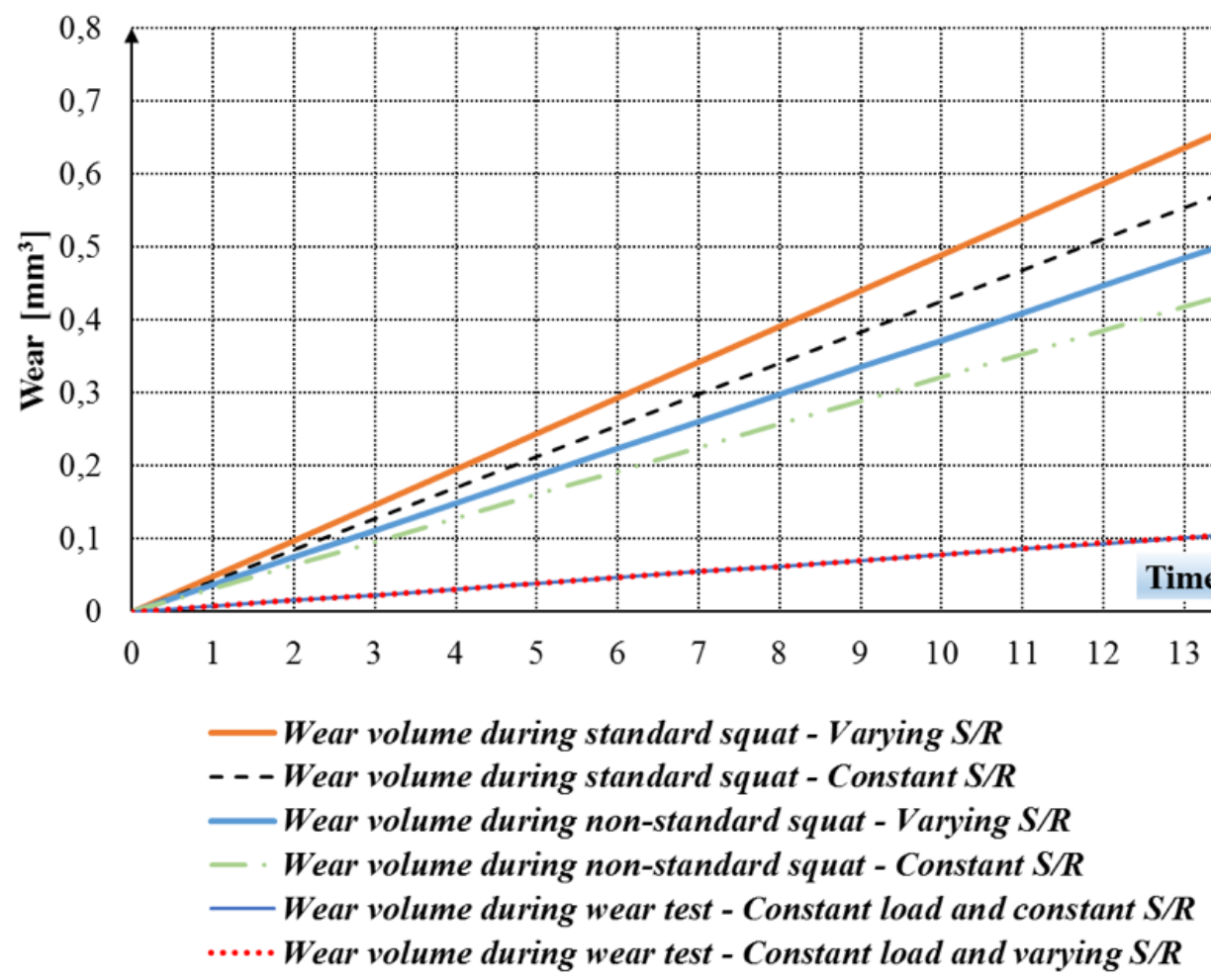

Figure 6. Total wear during lifetime cycle.

Figure 5 , the varying slide-roll ratio has also the effect of increasing the wear volume. A feasible explanation why wear volume is lower when constant 0.4 ( $40 \%$ of sliding) slide-roll is considered during squat can be addressed as follows: it was been proven by several studies, that during the flexion of the knee joint, approximately up to 20-30 degree of flexion, rolling is dominant ${ }^{11-12}$, which is not a crucial motion as far as wear is concerned, since the friction effect caused by sliding is also very low.

Therefore, the Authors who considered 0.3-0.4 slideroll ratio were correct with regard to beginning of the squat motion, but not with respect to the complete cycle. Above 30 degrees of flexion, as sliding prevails, wear starts developing between the surfaces due to the shear stress which is caused by the increasing slide ${ }^{22}$. Since the kinematic condition of the TKR geometries is different, wear must differ during the progression of the motion as well, which can be apparently observed on Figure 5.

The effect of the new parameters on wear, the slideroll ratio and the tibiofemoral force with and without the horizontal movement of the center of gravity, have been evidently demonstrated, and quantitatively determined. Varying slide-roll as a wear parameter causes approximately $15 \%$ more wear than the constant slide-roll ratio, regarding any type of squatting motion. The involvement of the tibiofemoral force has the major effect on wear, which can lead to $65 \%$ more removed volume per cycle compared to other configurations. As a further step, the augmented wear model is to be applied for other type of movements, such as gait and the cutting movement in football.

Limitations of the study must be pointed out as well. The friction force is calculated by the simple Coulomb-law, which assumes a point connection while in reality, the contact is a surface.

The specific wear rate $(k)$ can highly alter the phenomenon, and it can only be obtained via experiments for different kinematic conditions coupled with diverse materials. It should be studied further on, how well is it possible to estimate the correct $k$ value from the literature in order to gain adequate results from the mathematical analysis.

Furthermore, since the theoretical part of the model, with the additional biomechanical parameters, is established, the model can be applied as a wear-extension tool by incorporating it into a multibody model. By having a formidable numerical model ${ }^{12}$ created in the MSC.ADAMS, as a further step, the model could be used to analyze wear propagation in mobile bearing or mono-compartmental TKRs. This future model will be able to differentiate the wear between the lateral and medial compartments as well. Another future aim is to build a tribo-tester where the compressive force $\left(F_{t f}\right)$ can be adjusted according to the angle, thus the theoretical results, based on the new theory could be validated.

As for the clinical use of the results, two important findings can be concluded.

In one hand, it has been shown by the analytical wear model that during non-standard squat the total wear 
propagation is lower due to the special kinetics of this type of squat. This result has a relevant implication on rehabilitation: patients exercising non-standard squat, instead of standard squat where the torso does not lean forward, will have less impact on their implanted TKRs, with regard to wear, or furthermore even on their physiological knee joints as well.

On the other hand, it was mentioned that wear is only the $7^{\text {th }}$ cause of failure according to the study of Sharkey et al. ${ }^{2}$, while probably the most significant problem in TKRs is the loosening of the components. It must be highlighted, that some studies show that wear can also be the root cause of loosening ${ }^{23}$, due to the debris that are largely produced at the contacting surfaces. Wear debris has also the non-wanted effect on TKRs that they may induce biological reactions $^{25}$ like irritation in the soft tissue envelope and in the meniscus.

\section{Ethics}

The Authors declare that this research was conducted following basic ethical aspects and international standards as required by the journal and recently update $i^{26}$.

\section{Acknowledgements}

This work was supported by the ELTE Eötvös Loránd University in the frame of the ÚNKP-17-4 New National Excellence Program of the Ministry of Human Capacities, the National Natural Science Foundation of China (81301600) and Anta Sports Products Limited (HK2015000090), the Savaria Institute of Technology, Eötvös Loránd University and the Research Academy of Grand Health, Ningbo University.

\section{References}

1. Sanders AP, Lockard CA, Weisenburger JN, Haider H. Using a surrogate contact pair to evaluate polyethylene wear in prosthetic knee joints. J Biomed Mater Res A. 2016; 104:133-140.

2. Sharkey PF, Lichstein PM, Shen C, Tokarski AT, Parvizi J. Why are total knee arthroplasties failing today - Has anything changed after 10 years? J Arthroplasty. 2014;29:1774-1778.

3. Karlhuber M. Development of a method for the analysis of the wear of retrieved polyethylene components of total knee arthroplasty. Thesis, Technical University of Hamburg, Germany. 1995.

4. Guo Y, Hao Z, Wan C. Tribological characteristics of polyvinylpyrrolidone (PVP) as a lubrication additive for artificial knee joint. Tribol Int. 2016;93:214-219.

5. Qu M, Chyr A, Sanders AP, Raeymaekers B. Designing prosthetic knee joints with bio-inspired bearing surfaces. Tribol Int. 2014;77:106-110.

6. Wimmer MA, Andriacchi TP. Tractive forces during rolling motion of the knee: Implications for wear in total knee replacement. J Biomechanics. 1997;30:131-137.

7. Patten EW, Van Citters D, Ries MD, Pruitt LA. Wear of UHMWPE from sliding, rolling, and rotation in a multidirec- tional tribo-system. Wear. 2013;304:60-66.

8. Rawal BR, Yadav A, Pare V. Life estimation of knee joint prosthesis by combined effect of fatigue and wear. Procedia Tech. 2016;23:60-67.

9. McGloughlin T, Kavanagh A. The influence of slip ratios in contemporary TKR on the wear of ultra-high molecular weight polyethylene (UHMWPE): An experimental view. J Biomechanics. 1998;31:8.

10. Hollman JH, Deusinger RH, Van Dillen LR, Matava MJ. Knee joint movements in subjects without knee pathology and subjects with injured anterior cruciate ligaments. Phys. Ther. 2002;82:960-972.

11. Nägerl H, Frosch KH, Wachowski MM, Dumont $\mathrm{C}$, Abicht Ch, Adam P, Kubein-Meesenburg D. A novel total knee replacement by rolling articulating surfaces. In vivo functional measurements and tests. Acta Bioeng Biomech. 2008; 10:55-60.

12. Fekete G, De Baets $P$, Wahab MA, Csizmadia BM, Katona $G$, Vanegas-Useche LV, Solanilla JA. Sliding-rolling ratio during deep squat with regard to different knee prostheses. Acta Polytech Hung. 2012;9:5-24.

13. Laurent MP, Johnson TS, Yao JQ, Blanchard CR, Crowninshield RD. In vitro lateral versus medial wear of knee prosthesis. Wear. 2003;255:1101-1106.

14. Archard JF, Hirst W. The wear of metals under unlubricated conditions. Proc R Soc Lond A. 1956;236:397-410.

15. Pal S, Haider H, Laz PJ, Knight LA, Rullkoetter PJ. Probabilistic computational modeling of total knee replacement wear. Wear. 2008;264:701-707.

16. O'Brien ST, Bohm ER, Petrak MJ, Wyss UP, Brandt J-M. An energy dissipation and cross shear time dependent computational wear model for the analysis of polyethylene wear in total knee replacements. J Biomechanics. 2014;47:11271133.

17. Turell M, Wang A, Bellare A. Quantification of the effect of cross-path motion on the wear rate of ultra-high molecular weight polyethylene. Wear. 2003;255:1034-1039.

18. Abdelgaied A, Liu F, Brockett $C$, Jennings L, Fisher J, Jin Z. Computational wear prediction of artificial knee joints based on a new wear law and formulation. J Biomechanics. 2011;44:1108-1116.

19. Fekete G, Csizmadia BM, Wahab MA, De Baets P, Vanegas-Useche LV, Bíró I. Patellofemoral model of the knee joint under non-standard squatting. Dyna Colombia. 2014;81:6067.

20. Slater LV, Hart JM. Muscle activation patterns during different squat techniques. J Strength Cond Res. 2017;31:667-676.

21. Slater LV, Hart JM. The influence of knee alignment on lower extremity kinetics during squats. J Electromyogr Kinesiol. 2016;31:96-103.

22. Liu A, Jennings LM, Ingham E, Fisher J. Tribology study of natural knee using and animal model in a new whole joint natural knee simulator. J Bio-mechanics. 2015;48:30043011.

23. Brockett CL, Carbone S, Fisher J, Jennings LM. PEEK and CFR-PEEK as alternative bearing materials to UHMWPE in a fixed bearing total knee replacement: An experimental wear study. Wear. 2017;374-375:86-91.

24. Zhou Z, Jin Z. Biotribology: recent progresses and future perspectives, Biosurf. Biotribol. 2015;1:3-24.

25. Ellison P, Tipper JL, Jennings LM, Fisher J. Biological activity of polyethylene wear debris produced in the patellofemoral joint, Proc. Inst. Mech. Eng. Part H. 2012;226:377383.

26. Padulo J, Oliva F, Frizziero A, Maffulli N. Muscles, Ligaments and Tendons Journal - Basic principles and recommendations in clinical and field science research: 2016 update. MLTJ. 2016;6(1):1-5. 